\title{
Comparison of Fecal Egg Counts and ELISA for the diagnosis of Dicrocoelium Dendriticum infection
}

\author{
M. Naeemipour ${ }^{1}$, Gh.R. Hashemitabar ${ }^{2}$, K. Dastjerdi ${ }^{3}$, \\ M. Jamshidian Mojaver ${ }^{4}$, H.R. Mohammadi ${ }^{5}$
}

${ }^{1}$ Department of Biochemistry and Nutrition, Cellular and Molecular Research Center, Sabzevar University of Medical Sciences, Sabzevar, Iran

${ }^{2}$ Department of Pathobiology, Faculty of Veterinary Medicine, Ferdowsi University of Mashhad, Mashhad, Iran

${ }^{3}$ Department of Molecular Medicine, Cellular and Molecular Research Center, Birjand University of Medical Science, Birjand, Iran

${ }^{4}$ Mashhad Branch, Razi Vaccine and Serum Research Institute, Agricultural Research, Education and Extension Organization (AREEO), Mashhad, Iran

${ }^{5}$ Department of Clinical Sciences, Faculty of Veterinary Medicine, Semnan University, Semnan, Iran

\begin{abstract}
From economical point of view, Dicrocoelium Dendriticum (D. dendriticum) causes a lot of damages to the livestock industry annually. So, the rapid diagnosis of infection is very important. The diagnosis is based on egg per count of feces (EPG) test because detection according to clinical symptoms is difficult. Since EPG is not accurate and sensitive, the serological methods become important for the diagnosis of this parasite as they are more accurate in comparison to EPG test and they are able to diagnose infection in a short time. In this study, somatic and Excretory-secretory antigens (EsAg) were isolated. The ELISA test was set up according to positive and negative sera and the results which were obtained compared to those obtained by the EPG test. The prevalence of infection in 550 samples by ELISA and EPG methods were $56 \%$ and $7 \%$ respectively, which shows the significant difference between these methods in examining the rate of infection. Based on the results, the specificity and sensitivity in ELISA test were $95 \%$ and $94 \%$, respectively. The results showed that the ELISA is a more reliable test in comparison to EPG test for the rapid diagnosis of $D$. dendriticum infection.
\end{abstract}

Key words: Dicrocoelium dendriticum, ELISA, EPG, somatic antigen, Excretory-secretory antigen

Abbreviations: ELISA: enzyme-linked immunosorbent assay; SDS Page: Sodium dodecyl sulfate polyacrylamide gel electrophoresis; EPG: eggs per gram; EsAg: Excretory-secretory antigens; SoAg: Somatic antigens 


\section{Introduction}

D. dendriticum is one of the most prevalent liver trematodes in animals. This fluke lives inside the binary ducts of liver of cow, sheep, goat, pig and rarely in human (Rack et al. 2004, Jeandron et al. 2011, Beck et al. 2015, Ofori et al. 2015,). Anemia, Icterus, progressive liver cirrhosis, and weight loss are among the symptoms of being infected by this parasite (Sargison et al. 2012, Godara et al. 2014). The disease does not usually cause death, but the chronic infection incurs considerable economic losses to livestock industry, which includes a reduced production of meat, milk and wool. Also, a large number of infected livers are condemned (Jahed Khaniki et al. 2013).

Diagnosing dicrocoeliosis in small ruminants is difficult for many reasons, especially due to absence of symptoms of low infection. On the other hand, the damage caused by the parasite is significant. Hence the use of methods detecting antigens became routine tests, including the ELISA method. The diagnosis of parasite is done by EPG method which is not an accurate test and the rate of the diagnosis of the infected animals is very low (Ferre 1994). Since the number of eggs in feces is relatively low, the diagnosis of infection with low intensity is difficult or impossible (Otranto and Traversa 2002). So an appropriate serology method should be used to examine the rate of infection. One of the most common tests is the ELISA. This method has many advantages: The presence of antibodies against the parasite can be examined in sheep blood four weeks after the infection (Gonzalez-Lanza et al. 2000), it is simple and a large number of samples can be tested in a short time, the method is highly accurate and efficient. It is used in epidemiological studies (Sanchez-Andrade et al. 2003, Broglia et al. 2009, Colwell and Goater 2010, Arias et al. 2012).

Dicrocoelium is one of the most important parasite that should be detected as soon as possible with respect to rapid diagnosis and treatment of the infection reduce the economic damages. In this study, we developed an indirect ELISA for detection of Dicrocoelium in sheep and compared the results with the EPG test.

\section{Materials and Methods}

Animals: Blood and fecal samples were randomly obtained from 550 sheep (151 male and 382 female), 1 to 10 years of age from farms in the suburbs of Mashhad, Iran. Positive samples included 50 sheep, 5 years to 8 years of age, 18 male and 32 female. All negative lambs were male and 1-2 months of age.
Excretory-secretory antigen: Excretory-secretory (EsAg) antigens from D. dendriticum adult worms were obtained as previously described (Gonzalez-Lanza et al. 2000) with some modifications. Freshly matured worms were removed from the liver bile ducts of sheep obtained at a local abattoir. The collected worms were washed in phosphate-buffered saline (PBS pH 7.4) with gentamycin (40 mg/l Sigma) at $37^{\circ} \mathrm{C}$ three times. Then, the live parasites were cultured in RPMI 1640 medium (Biosera, Boussens, France) supplemented with $200 \mathrm{mM}$ N-acetyl-L-alanil-L-glutamine (Sigma), $0.3 \mathrm{~g} / \mathrm{l}$ sodium bicarbonate $7.5 \%$ (Sigma) and $40 \mathrm{mg} / 1$ gentamycin at $37^{\circ} \mathrm{C}$ for $48 \mathrm{~h}$. Worm viability was checked on the basis of motility of the worms observed under a stereomicroscope. After removing the parasites, medium containing EsAg products was collected and centrifuged at $10,000 \mathrm{~g}$ for $15 \mathrm{~min}$ at $4^{\circ} \mathrm{C}$. The supernatant was filtered through $0.22 \mu \mathrm{m}$ pore size filter units and then a cocktail of protease inhibitors (Sigma) was added. The fluid was finally concentrated by YM-50 membranes (50,000 molecular weight cut-off). Protein concentration was determined by the Bradford method (Bradford 1976). Samples were aliquoted and stored at $-80^{\circ} \mathrm{C}$ until used.

Somatic antigen: Adults of $D$. dendriticum were washed several times in PBS pH 7.4. Approximately 200 washed flukes were placed in $8 \mathrm{ml}$ PBS with the following protease inhibitors: EDTA (Merck) $1 \mathrm{mM}$, N-ethylmaleimide (Merck) $1 \mathrm{mM}$, phenyl methyl sulfonyl fluoride (Merck) $1 \mathrm{mM}$ and Pepstatin A (Merck) $0.1 \mathrm{~lm}$. Then, they were sonicated by an ultrasound processor with pulse-type vibration for $1 \mathrm{~min}$ and 3 pulses of $30 \mathrm{sec}$ each. Finally, the suspension was centrifuged at $10,000 \mathrm{~g}$ for $30 \mathrm{~min}$ and the supernatant fraction collected (Gonzalez-Lanza et al. 2000). After estimating the protein concentration by the method of Bradford, the antigen was stored in small aliquots at $-80^{\circ} \mathrm{C}$ until used.

Antibody detection by indirect ELISA: Antigenic bands purified by electroelution and plates were coated with pure antigen (Es or So) in $\mathrm{NaHCO} 3 / \mathrm{Na} 2 \mathrm{CO} 3$ (Merck) buffer pH 9.6 at 100 $\mu \mathrm{l} /$ well and then incubated overnight at $4^{\circ} \mathrm{C}$. After removal of the antigen, the plates were washed three times with PBS containing 0.05\% Tween 20 (PBST) (Merck) for $5 \mathrm{~min}$ each. The unbound sites in each well were blocked by incubation in a humidity chamber for $1 \mathrm{~h}$ at $37^{\circ} \mathrm{C}$ with $100 \mu \mathrm{l} 5 \%$ skimmed milk diluted in PBST. Then, $100 \mu \mathrm{l}$ sera from infected sheep were added and incubated for $2 \mathrm{~h}$ at $37^{\circ} \mathrm{C}$. After incubation, the plates were washed three times with PBST. All sera were tested in duplicate. Then $100 \mu \mathrm{l}$ anti-sheep IgG peroxidase conjugate (Jackson) (diluted to 1:5,000 in PBST) was added to each well. 
The plates were incubated for $1 \mathrm{~h}$ at $37^{\circ} \mathrm{C}$ and then washed three times as previously described. After a new wash, $100 \mu \mathrm{l}$ of a solution of 3, 3, 5, 5' - tetramethylbenzidine (TMB) (Sigma) was added to each well and the plates were incubated for $15 \mathrm{~min}$ in darkness at room temperature. The color reaction was stopped by the addition of $100 \mu \mathrm{l} 1 \mathrm{~N}$ sulfuric acid (Merck) and optical density values were read at 450 $\mathrm{nm}$ using an Automated Microplate Reader Elx800 (Bio-Tek Instruments, USA) . Three controls were used on each plate: (1) a negative control pool serum sample from sheep negative to $D$. dendriticum, (2) a positive control serum sample from a sheep highly infected with $D$. dendriticum and (3) a conjugate control in which the serum was substituted by PBST. Each serum sample was tested in duplicate and the results were expressed as the mean of the OD values obtained. For antibody detection we considered positive those samples in which mean OD values exceeded the mean of negative controls plus three standard deviations of that mean (the cut-off point). Twenty sheep were used as negative controls. The data were analyzed by ANOVA and Tukey's multiple comparison test.

SDS-PAGE analysis: SDS-PAGE of somatic and excretory/secretory proteins $(30 \mu \mathrm{g})$ was performed in different percentage polyacrylamide gels using a discontinuous system as the concentration of acrylamide was $12.5 \%$. For size estimation, a pre-stained protein standard broad range (10-170 kDa) (Fermentas) was also subjected to electrophoresis, which was carried out using a Mini-Cell (Bio-Rad) at $70 \mathrm{~V}$ constant voltage for $3 \mathrm{~h}$. Proteins were stained with Coomassie Blue (Fermentas) (Revilla-Nuin et al. 2005).

Western blot analysis: immunoblotting was used to reveal which antigen has immunized animals. Small aliquots $(10 \mu \mathrm{l})$ of extracts were reserved for protein determination using Bradford assay reagent from Fermentas (Fermentas, USA). Proteins were separated by SDS-poly acrylamide electrophoresis in slab gels of $12.5 \%$ poly acrylamide, made in duplicate and transferred to nitrocellulose membranes (Problat MN 0.45 $\mathrm{mm}$, Takara, Otsu, Japan). Afterwards, the transferred proteins were blocked with blocking solution (Tris-buffered saline, TBS, containing 5\% skimmed milk) at room temperature for 30-60 min. Membranes were washed $(2-5 \mathrm{~min})$ in TBST (TBS with $0.05 \%$ Tween-20) and incubated for 1-2 h with anti- $D$. dendriticum sheep sera. The membranes were washed again (2-5 min) in TBS and developed with rabbit anti-sheep $\mathrm{IgG}$ peroxidase (Jackson) conjugate for $1 \mathrm{~h}$ in the dark at room temperature. Another 2-3 washes were carried out. All incubations were performed with shaking. After evaluating different quantities of proteins and several dilutions of both primary and secondary antibodies, we established as optimal conditions: $40 \mu \mathrm{g}$ of antigen protein by lane for SDS-PAGE; $1 / 50$ dilution of the anti- $D$. dendriticum or other parasite sheep sera and 1/500 dilution of the rabbit anti-sheep IgG for Western blot analysis. Antibody binding was revealed by incubation with horseradish peroxidase-conjugated secondary antibody rabbit anti-sheep IgG $(\mathrm{H}+\mathrm{L})$ peroxidase conjugated (Pierce Biotechnology Inc., USA) for $1 \mathrm{~h}$ in the dark at room temperature. The ECL Plus immunoblotting detection system (GE Healthcare Biosciences, Buckinghamshire, UK) was used to detect HRP activity on a chemiluminescence detector system (G Box, Syngene, Cambridge, England). (Revilla-Nuin et al. 2005).

Egg per gram of feces test: The egg per gram of feces (EPG) test was performed by the McMaster technique. In each case to assess the level of infection, $4 \mathrm{~g}$ of feces was mixed in $56 \mathrm{ml}$ of tap water, and the sample was mixed with a stirring device. The faecal suspension was left for $30 \mathrm{~min}$ at room temperature. The suspension was poured through a tea strainer into a clean new container, and a $10-\mathrm{ml}$ tube was filled to capacity with the filtered suspension. The tube was centrifuged for $5 \mathrm{~min}$ at $1200 \mathrm{rpm}$ and the supernatant was removed. Shortly before commencing the egg count, $4 \mathrm{ml}$ of flotation fluid (saturated $\mathrm{NaCl}$ with $500 \mathrm{~g}$ glucose per litre of water) was added to a centrifuge tube. The sediment was then carefully resuspended and both sides of the McMaster counting chamber were filled. The filled McMaster chamber was left for 3-5 min to rest before counting. The EPG was obtained by multiplying the total number of eggs counted in the two squares of the counting chambers of the McMaster slide. Helminth eggs present were identified using standard parasitological criteria (Anon 1977, Roepstorff and Nansen 1998).

\section{Results}

Detection of antigenic polypeptides: The concentration of the Somatic and EsAg antigens was $2 \mu \mathrm{g} / \mu \mathrm{l}$ and $0 / 4 \mu \mathrm{g} / \mu \mathrm{l}$, respectively. In order to reach the concentration of the EsAg to $1 \mu \mathrm{g} / \mu \mathrm{l}$, the samples were concentrated. $40 \mu \mathrm{g}$ each antigen was run on $12 / 5 \%$ gel and was stained by Coomassie Blue (suppl. Fig. 1). In order to find immunogenic antigens, antigens were transferred to nitrocellulose membrane and western blot was done. Two antigenic bands with the size of 67 and $130 \mathrm{kDa}$ were identified (Fig. 1). In order to examine the cross reaction with Fasciola hepatica and verify the affiliation of related bands to Dicrocoelium, anti-fasciola antibodies were used and 


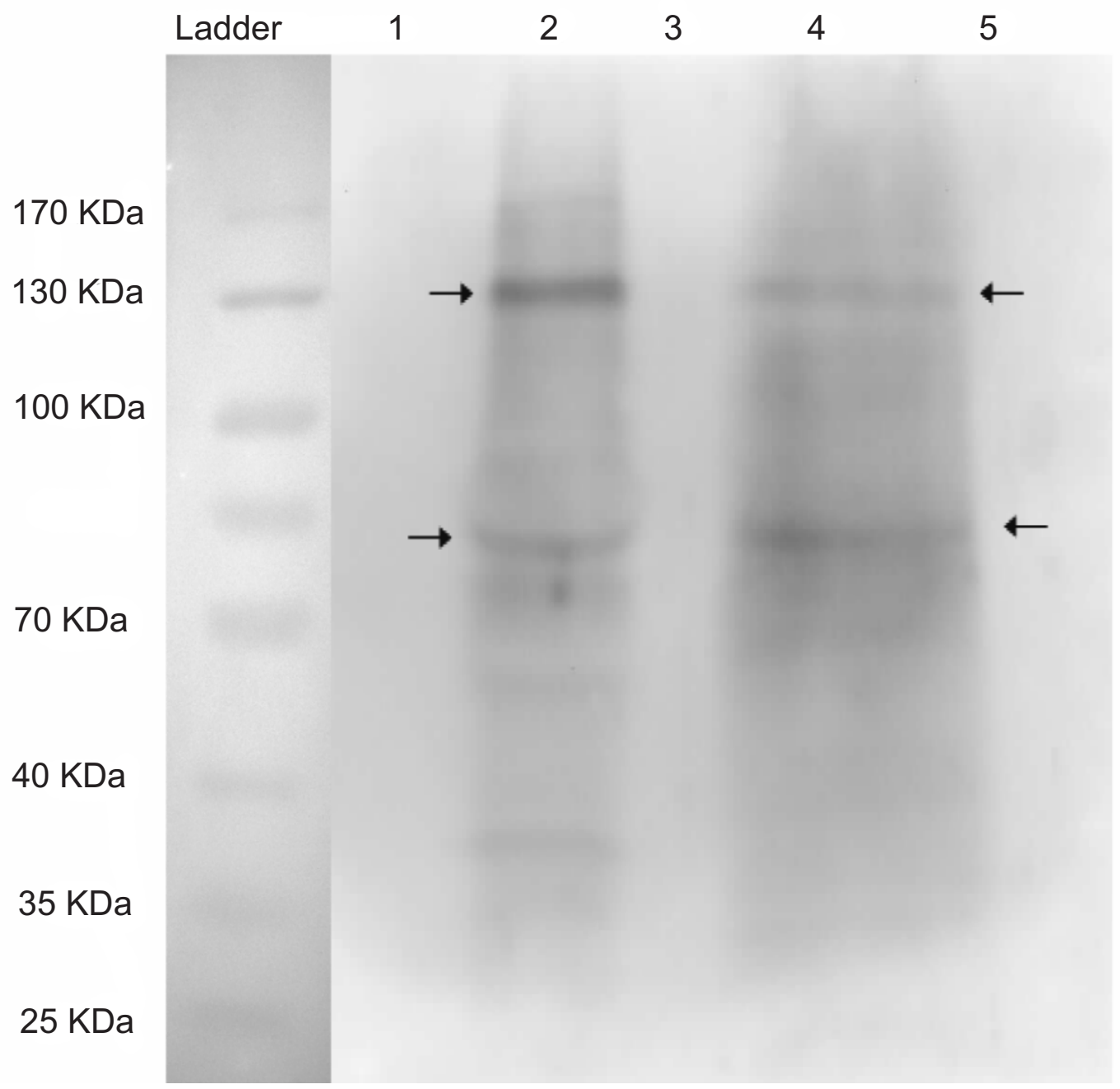

Fig. 1. Detected bands in the sera of sheep positive for $D$. dendriticum by Western blotting. Lane 1. Used of anti-fasciloa hepatica serum with somatic antigen. Lane 2, somatic antigen with anti- D. dendriticum serum. Lane 3, Used of anti-fasciloa hepatica serum with exceratory antigen. Lane 4 , exceratory antigen with anti- $D$. dendriticum serum. Lane 5 , antigen without use anti-sera.

it was confirmed that the two identified bands were specific to Dicrocoelium (Fig. 1).

Evaluation by indirect ELISA: In ELISA, the higher the purity of the coated antigens, the higher the sensitivity of the test and a more accurate diagnosis will become possible. In this study, the EsAg and somatic antigens were first run by SDS-page gel and immunogenic antigens were identified by western blot. These antigens were coated in ELISA microplates. 50 positive control samples and 20 negative control samples were used to set up the indirect ELISA. Positive samples were chosen from infected livers in abattoir, which were only infected by $D$. dicrocoelium. The negative samples were chosen from uninfected lambs. From 50 positive samples, 47 samples were positive with ELISA (95\% CI=0.891) (Fig. 2) and from 20 negative samples, 19 were negative (95\% CI=0.666) (Fig. 3). We divided 50 sheep into two groups; low and high level of infection according to investigation of liver in laboratory. In low level of infection, no sample were detected by EPG method but 11 samples were positive by ELISA. In high level of infection, only four samples were positive with EPG whereas 36 samples were detected with ELISA (Table 1). This means that ELISA method is more sensitivity test than EPG method. The sensitivity and specificity of the ELISA test was 94\% and 95\% respectively, which is considered to be high in comparison to other studies.

Compare ELISA and EPG techniques: The results obtained from ELISA method were compared to those from the EPG test (Table 2). Among 550 randomly chosen samples, there were 308 positive and 242 negative in ELISA, while there were 39 positive and 511 negative samples in the EPG method (Table 2 ). This corresponded to $56 \%$ prevalence of the infection with ELISA method and 7\% with EPG method (Fig. 4). 


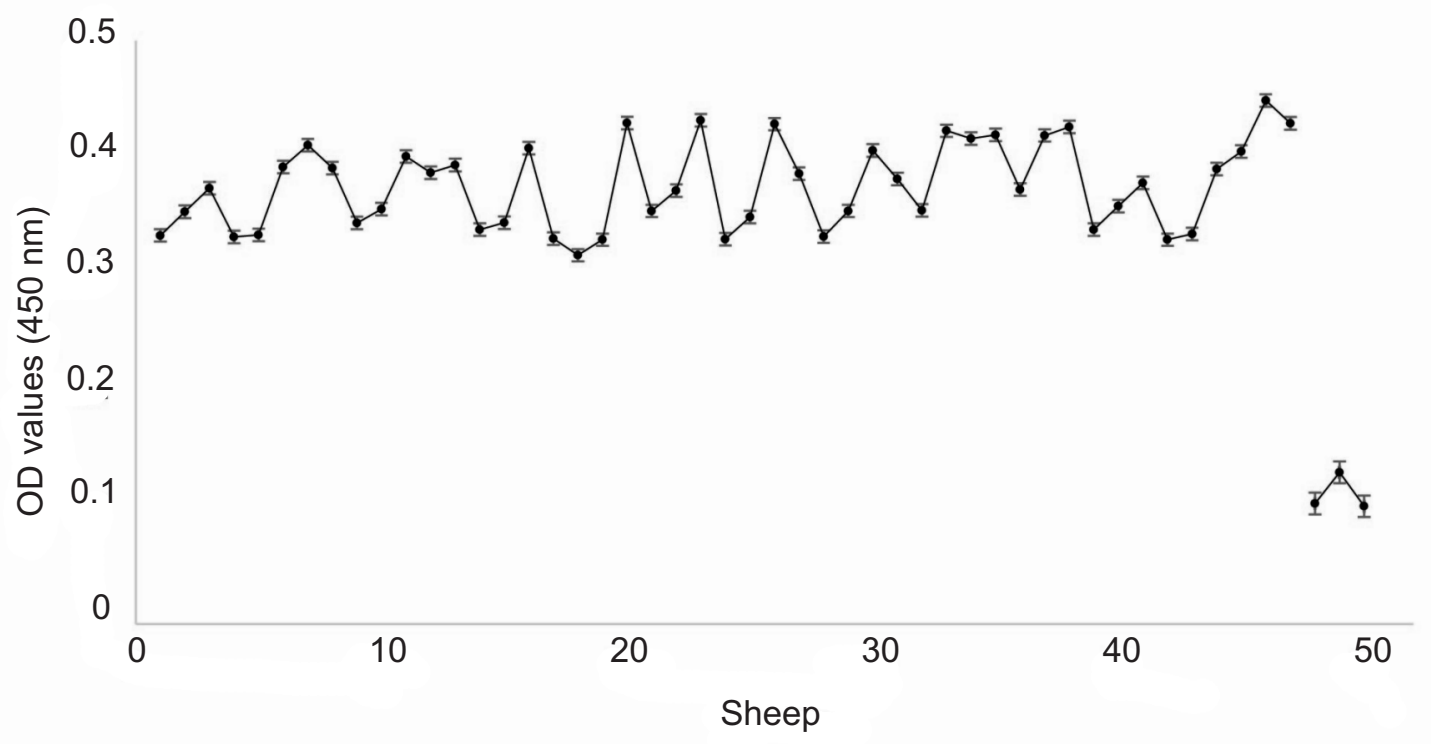

Fig. 2. Optical Density (OD) values obtained by indirect ELISA technique using positive sheep sera (Cut-off: 0.2008).

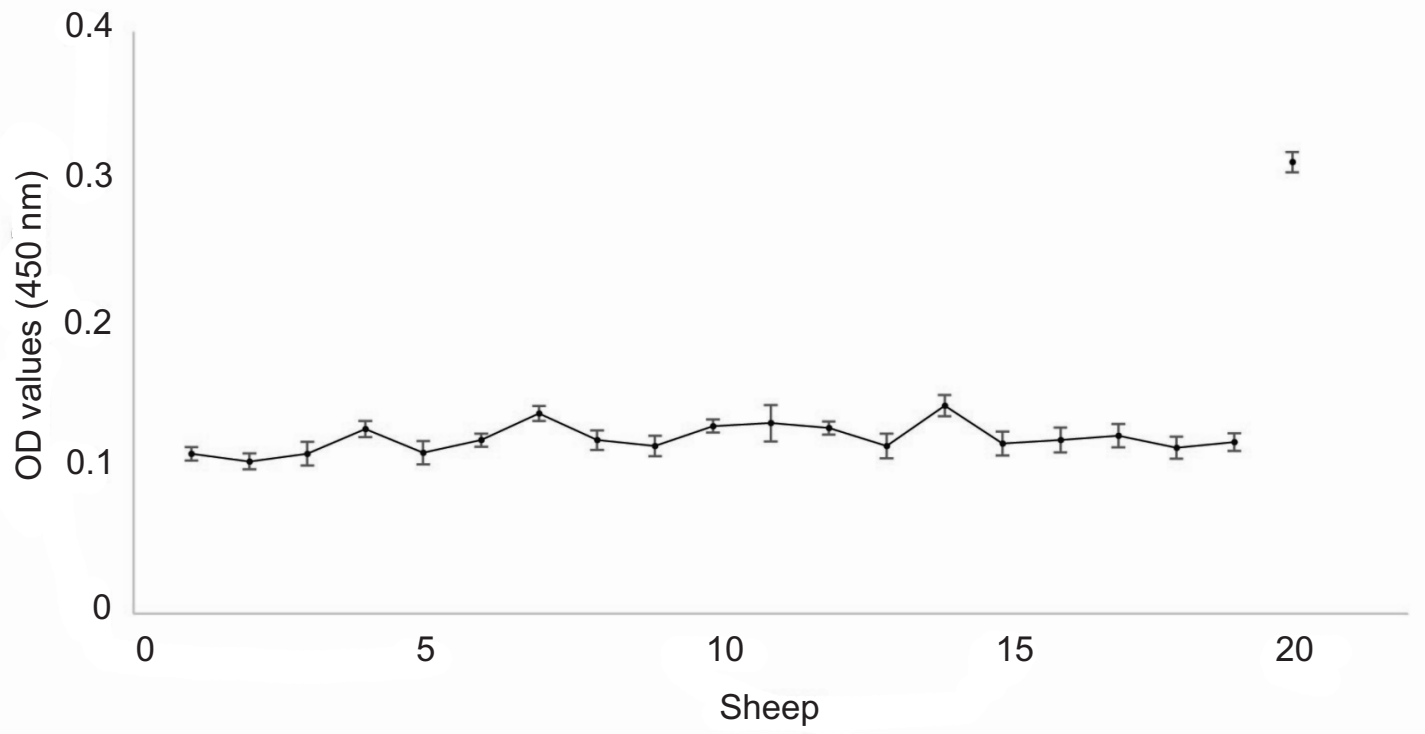

Fig. 3. Optical Density (OD) values obtained by indirect ELISA technique using negative sheep sera (Cut-off: 0.2008).

Table 1. Egg-output and seropositivity in the 50 sheep positive for Dicrocoelium dendriticum.

\begin{tabular}{lcccc}
\hline & Number of sheep & Faecal-output & Elisa & Not detected by Elisa \\
\hline Low level of infection & 14 & 0 & 11 & 3 \\
\hline High level of infection & 36 & 4 & 36 & 0 \\
\hline Total & 50 & $4^{*}$ & 47 & 3 \\
\hline
\end{tabular}

* also detected by Elisa 
70

60

50

40

30

20

10

0

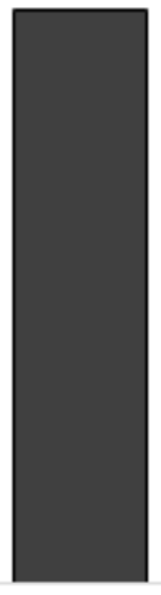

Sheep/Elisa

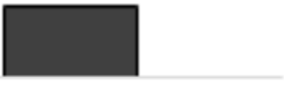

Sheep/Egg

Fig. 4. Prevalences (\%) of D. dendriticum egg-output and serum antibodies in 550 sheep.

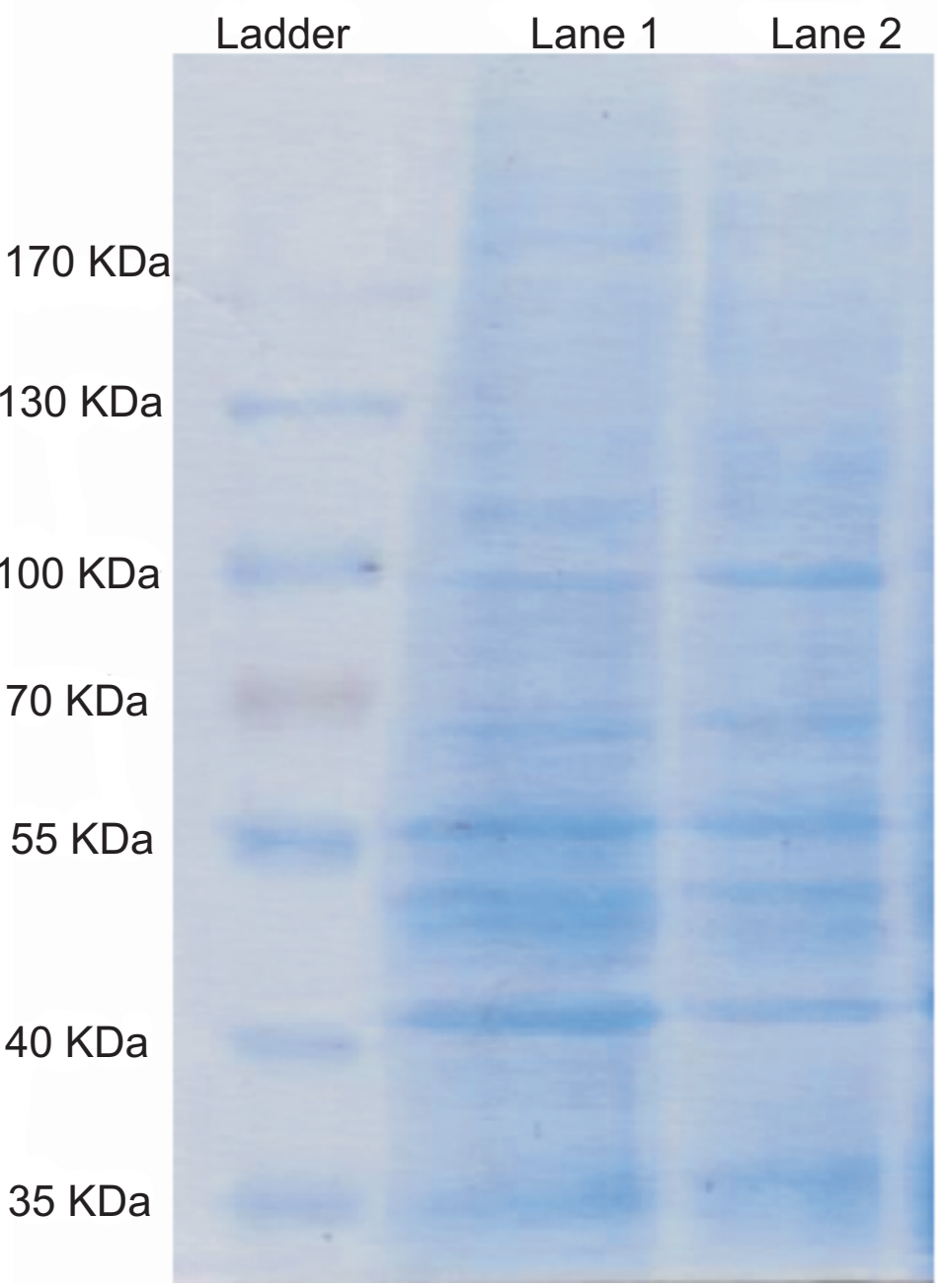

Supplementary Figure 1: Detected protein bands in somatic antigen (lane 1) and EsAg of Dicrocoelium dendriticum using SDS-PAGE and staining with coomassie blue. 
Table 2. Dicrocoelium dendriticum egg-output and seropositivity in 550 sheep.

\begin{tabular}{lccc}
\hline & Groups & Number of sheep & Total \\
\hline Faecal output & Detected & $39^{*}$ & 550 \\
\cline { 2 - 4 } & Not detected & 511 & 550 \\
\cline { 2 - 4 } & Detected & 308 & 550 \\
\cline { 2 - 4 } Gender & Not detected & 242 & 152 \\
\hline Both techniques & male & 398 & 550 \\
\cline { 2 - 4 } & female & 308 & 242 \\
\hline
\end{tabular}

* also detected by Elisa

\section{Discussion}

The present study was designed with the purpose of using indirect ELISA method with the help of purified antigens in diagnosing D. dicrocoelium in sheep, detecting the prevalence of infection, and comparing this method with EPG. Definitive diagnosis of Dicrocoelium, because of imprecise symptoms and shared symptoms with other parasites, needs laboratory confirmation (Khandelwal et al. 2008). Different methods such as EPG and serology methods have been used for diagnosis so far (Calamel 1977, Hiepe 1994, Sek et al. 2004, Revilla-Nuin et al. 2005). The EPG method is a routine procedure which is not able to show the precise level of infection, difficult to work with, and also very time consuming. On the other hand, many factors affect egg excretion of the parasites and the number of eggs per gram of feces. The amount of egg excretion changes with the season. In winter, this amount is lower in comparison to spring. Even the amount of egg excretion changes in different months or even during a single day. In the case of Dicrocoelium, the excretion of eggs is higher in the afternoon than in the morning (Theodoridis et al. 1991). The diet and the use of anti-parasite medicine also affect egg excretion. Depending on the number of parasite, the intensity of egg excretion will be different. The higher the number of parasites, the greater the amount of egg excretion (Manga-Gonzalez \& Gonzalez-Lanza 2005). According to the parasite life stage, it takes a long time for the egg to appear in feces. In a study done by Campo et al. (1999), the greatest amount of excreted eggs was found 150 to 180 days after the infection. All these factors make the EPG method not convenient and unreliable and necessitate the use of serology methods. Serological methods are of significant importance due to their simplicity, cheapness, and accuracy. So far, different serological methods have been used for diagnosis of Dicrocoeliosis. Among them, ELISA has been most extensively studied as it allows simultaneous examination of a big number of samples in a short time. Also, it is highly sensitive in rapid diagnosis of the disease. According to a study by Bode and Geye (1981) in which six different serological methods were used to diagnose Dicrocoelium in experimentally infected hamsters, the complement fixation and ELISA showed the highest sensitivity in rapid diagnosis of antibodies against Dicrocoelium. In a study by Savitskii et al. (1983) on different serological methods used for diagnosis of Dicrocoelium, the passive haemagglutination and ELISA tests had the highest specificity. The study done in sheep infected experimentally (Gonzalez-Lanza et al. 2000), the antibodies against Dicrocoelium could be identified after 30 days using indirect ELISA. This immunity refers to the migration of premature parasites to the liver and the highest amount of antibodies was 60 days after infection which persisted up to 180 days.

In a study by Simsek et al. (2004), the sensitivity of the test was calculated to be $86 \%$, but the specificity was low. In the present study, the sensitivity and specificity of the ELISA test were high: $94 \%$ and $95 \%$, respectively which may be due to the fact that we used purified antigens for the ELISA test. We identified two immunogenic bands (67 and $130 \mathrm{kDa}$ ) and after purification these antigens were used to set up the ELISA test, while in a study by Revilla et al. (2005), only one immunogenic antigen band was identified.

Most epidemiologic studies in Iran have used the EPG test and this is the first study which uses the indirect ELISA test for examining the level of infection with this parasite. The infection prevalence was $56 \%$ which shows that the studies areas are highly infected by Dicrocoelium parasite. Therefore, the ELISA test is a precise and reliable method for examining the prevalence of infection. With ELISA, infection can be diagnosed quickly and with treatment, economic damages can be prevented. In contrary, EPG is not a precise test and it is not suitable 
for epidemiologic studies especially with at low level of infection.

\section{Acknowledgements}

Authors would like to thank Faculty of Veterinary Medicine, Ferdowsi University of Mashhad, Iran for the grant they provided for completion of this project. We would also like to thank the Department of Biochemistry and Nutrition, Cellular and Molecular Research Center, Sabzevar University of Medical Sciences, Sabzevar, Iran for cooperation.

\section{References}

Anon (1977) Manual of veterinary parasitological laboratory techniques. Technical Bulletin No. 18. London.

Arias MS, Martinez-Carrasco C, Leon-Vizcaino L, Paz-Silva A, Diez-Banos P, Morrondo P, Alonso F (2012) Detection of antibodies in wild ruminants to evaluate exposure to liver trematodes. J Parasitol 98: 754-759.

Beck MA, Goater CP, Colwell DD (2015) Comparative recruitment, morphology and reproduction of a generalist trematode, Dicrocoelium dendriticum, in three species of host. Parasitology 43: 1-9.

Bode L, Geyer E (1981) Experimental dicrocoeliasis: the humoral immune response of golden hamsters and rabbits to primary infection with Dicrocoelium dendriticum. Z Parasitenkd 66: 167-178.

Bradford MM (1976) A rapid and sensitive method for the quantitation of microgram quantities of protein utilizing the principle of protein-dye binding. Anal Biochem 72: 248-254.

Broglia A, Heidrich J, Lanfranchi P, Nockler K, Schuster R (2009) Experimental ELISA for diagnosis of ovine dicrocoeliosis and application in a field survey. Parasitol Res 104: 949-953.

Calamel M (1977) Application of the technique immunofluorescence, indirect epidemiological study of dicrocoeliose. Rec Med Vet 153: 343-348.

Campo R, Manga-Gonzalez MY, Gonzalez-Lanza C (1999) Relationship between egg output and parasitic burden in lambs experimentally infected with different doses of Dicrocoelium dendriticum. Vet parasitol 87: 139-149.

Colwell DD, Goater CP (2010) Dicrocoelium dendriticum in cattle from Cypress Hills, Canada: humoral response and preliminary evaluation of an ELISA. Vet parasitol 174: 162-165.

Ferre I, Ortega-Mora LM, Rojo-Vazquez FA (1994) Prevalence of Dicrocoelium dendriticum infection in Leon province (NW Spain). Prev Vet Med 21: 147-154.
Godara R, Katoch R, Yadav A, Borah MK (2014) Dicrocoeliosis in goats in Jammu, India. J Parasit Dis 38: 201-204.

Gonzalez-Lanza C, Manga-Gonzalez MY, Campo R, Del-Pozo P, Sandoval H, Oleaga A, Ramajo V (2000) IgG antibody response to ES or somatic antigens of Dicrocoelium dendriticum (Trematoda) in experimentally infected sheep. Parasitol Res 86: 472-479.

Hiepe T (1994) Helminth control in sheep and goat flocks. Tierarztl Prax 22: 29-34.

Jahed Khaniki GR, Kia EB, Raei M (2013) Liver condemnation and economic losses due to parasitic infections in slaughtered animals in Iran. J Parasit Dis 37: 240-244.

Jeandron A, Rinaldi L, Abdyldaieva G, Usubalieva J, Steinmann P, Cringoli G, Utzinger J (2011) Human infections with Dicrocoelium dendriticum in Kyrgyzstan: the tip of the iceberg. J Parasitol 97: 1170-1172.

Khandelwal N, Shaw J, Jain MK (2008) Biliary parasites: diagnostic and therapeutic strategies. Curr Treat Options Gastroenterol 11: 85-95.

Manga-Gonzalez MY, Gonzalez-Lanza C (2005) Field and experimental studies on Dicrocoelium dendriticum and dicrocoeliasis in northern Spain. J Helminthol 79: 291-302.

Ofori M, Bogoch, II, Ephraim RK (2015) Prevalence of Dicrocoelium dendriticum ova in Ghanaian school children. J Trop Pediatr 61: 229-230.

Otranto D, Traversa D (2002) A review of dicrocoeliosis of ruminants including recent advances in the diagnosis and treatment. Vet Parasitol 107: 317-335.

Rack J, Adusu E, Jelinek T (2004) Human infection with Dicrocoelium dendriticum. Dtsch Med Wochenschr 129: 2538-2540.

Revilla-Nuin B, Manga-Gonzalez MY, Minambres B, Gonzalez-Lanza C (2005) Partial characterization and isolation of $130 \mathrm{kDa}$ antigenic protein of Dicrocoelium dendriticum adults. Vet Parasitol 134: 229-240.

Roepstorff A, Nansen P (1998) Epidemiology, diagnosis and control of helminth parasites of swine. FAO Animal Health Manual, Rome.

Sanchez-Andrade R, Paz-Silva A, Suarez JL, Arias M, Lopez C, Morrondo P, Scala A (2003) Serum antibodies to Dicrocoelium dendriticum in sheep from Sardinia (Italy). Prev Vet Med 57: 1-5.

Sargison ND, Baird GJ, Sotiraki S, Gilleard JS, Busin V (2012) Hepatogenous photosensitisation in Scottish sheep casued by Dicrocoelium dendriticum. Vet Parasitol 189: 233-237.

Savitskii IG (1983) Immunological investigations of sera during experimental dicrocoeliasis in rabbit with different antigens of Dicrocoelium dendriticum. Byull Vses Inst Gel Mintol 82: 35-66.

Simsek S, Ceribazi AO, Utuk AE (2004) Morphological and Histopathological Examination of Liver Damage Caused by Dicrocoelium dendriticum in Sheep Livers. Turkiye Parazitol Derg 28: 189-191.

Theodoridis Y, Duncan JL, MacLean JM, Himonas CA (1991) Pathophysiological studies on Dicrocoelium dendriticum infection in sheep. Vet Parasitol 39: 61-66. 